\title{
Susceptibility of different phases of biofilm of Klebsiella pneumoniae to three different antibiotics
}

\author{
Saloni Singla, Kusum Harjai and Sanjay Chhibber
}

The existence of majority of bacteria in biofilm mode makes it difficult to eradicate them as antibiotics at much higher concentrations than the MICs are required to destroy these bacteria. This study investigated the effect of different classes of antibiotics on different phases of biofilm formed by Klebsiella pneumoniae. The organism was grown in different phases relevant to biofilm formation: planktonic cells at mid-log phase, planktonic cells at stationary phase, adherent monolayers and mature biofilms and their susceptibility to different classes of antibiotics was assessed. The results showed that planktonic organisms were susceptible to ciprofloxacin, amikacin and piperacillin, and their MBC values were same or eight times higher than their corresponding MICs. MBC of ciprofloxacin and amikacin was found to be four and eight times higher for monolayer than planktonic cells. On the other hand, MBC of piperacillin was $>1024 \mu \mathrm{g} \mathrm{ml}^{-1}$. K. pneumoniae in a biofilm growth mode was more resistant to antibiotics than all other modes. The effect of amikacin and ciprofloxacin on young and older biofilms, at the highest achievable serum concentrations, was also examined. It was observed that amikacin at a concentration of $40 \mu \mathrm{g} \mathrm{ml}^{-1}$ was able to eradicate the young biofilms; however, with increase in the age of the biofilm, it became completely ineffective. Calcofluor staining suggested increased production of exopolysaccharide in older biofilm compared with younger biofim that might be responsible for the increased resistance of older biofilm of $K$. pneumoniae to antibiotics. The Journal of Antibiotics (2013) 66, 61-66; doi:10.1038/ja.2012.101; published online 21 November 2012

Keywords: amikacin; antibiotic resistance; biofilm; Klebsiella pneumonia

\section{INTRODUCTION}

Klebsiella pneumoniae is an opportunistic pathogen that is commonly associated with urinary tract infections (UTI), pneumonia, septicemia and wound infections. K. pneumoniae forms biofilm on indwelling plastic devices, such as catheters and endotracheal tubing, and subsequently colonize human tissue. One of the early stages of K. pneumoniae airway infections may also involve biofilm formation. ${ }^{1}$ The capsule and fimbriae are prominent structural components of the $K$. pneumoniae cell surface and have a significant role in biofilm formation. The mature biofilm thus formed is believed to cause chronic infections. Over $60 \%$ of bacterial infections and up to $80 \%$ of chronic infections are currently considered to involve microbial growth in biofilms. ${ }^{2}$ The increased antibiotic resistance of biofilm cells can be up to 1000 -fold greater than for free-living, planktonic bacterial cells. ${ }^{3}$ Resistance to antimicrobials can be attributed to several factors such as permeability barrier imposed by the aggregative biopolymers that hold the biofilm together ${ }^{4}$ and the existence of microbial cells in stationary phase. ${ }^{5}$ Biofilm age is also known to influence the outcome of a therapeutic regimen.

For chronic biofilm infections, no regimens for antibiotics have been established because the susceptibility of bacteria growing in biofilms differs markedly from planktonic cells in the same environment. Thus, killing dose of an antibiotic determined by Clinical and Laboratory Standards Institute method for planktonic bacteria has no effect on bacteria contained within a biofilm, even though cells shed from the biofilm may be eradicated. ${ }^{6}$ The biofilm MIC can be used to prevent febrile phase of infections by inhibiting planktonic cells released from biofilms. ${ }^{6,7}$ MIC for planktonic bacteria may or may not be similar to biofilm MIC, which depends on the class of antibiotics. ${ }^{6-8}$ The minimum biofilm eradication concentration, which targets complete bacterial killing, would be more meaningful than the biofilm MIC to prevent chronic phase of biofilm infections, which are characterized by less active dispersal. ${ }^{3,6}$ The minimum biofilm eradication concentration is impractical to achieve when conventional antibiotics are used in vitro, ${ }^{9,10}$ but the biofilm MBC, which targets $99.9 \%$ killing of biofilm-grown bacteria, may be achievable.

To our knowledge, no information is available about the comparative study where the effect of different classes of antibiotics has been studied on different phases of biofilm formation (planktonic cells at mid-log phase, planktonic cells at stationary phase, adherent monolayers and mature biofilms) by K. pneumoniae. In this study, the effect of antibiotics at peak serum achievable concentrations was studied on different phases of biofilm formed by K. pneumoniae. 


\section{MATERIALS AND METHODS}

\section{Bacterial strain and media}

Klebsiella pneumoniae B5055 (O1:K2) obtained originally from Dr M Trautman (Department of Medical Microbiology and Hygiene, University of Ulm, UIm, Germany) was used in this study. All antimicrobial susceptibility experiments were performed in soyabean casein digest medium. Growth media and antibiotics were purchased from HiMedia Laboratories (Mumbai, India). Nutrient agar was used as the culture medium for K. pneumoniae and bacterial dilutions were made in sterile $0.85 \%$ normal saline (NS).

\section{Antibiotics}

Piperacillin, amikacin sulfate and ciprofloxacin hydrochloride representing members of $\beta$-lactam, aminoglycoside and fluoroquinolone classes, respectively, purchased from HiMedia Laboratories was used.

\section{Development of adherent monolayers}

Bacterial cultures of adherent monolayers were established by the modified method of Miyake et al. ${ }^{11}$ Briefly, wells of microtiter plate were inoculated with $50 \mu \mathrm{l}$ of soyabean casein digest medium and $50 \mu \mathrm{l}$ of bacterial culture $\left(\mathrm{OD}_{600}=0.3\right)$ equivalent to $10^{8} \mathrm{CFU} \mathrm{ml}{ }^{-1}$ of $\mathrm{K}$. pneumoniae and incubated at $37^{\circ} \mathrm{C}$ for $1 \mathrm{~h}$. After incubation, planktonic bacteria in the fluid were removed and wells were washed thoroughly three times with NS. Adherent monolayers formed on the well surface was scraped using a sterile spatula. Scraped biofilm was suspended in $100 \mu \mathrm{l}$ NS and vortexed three times for 1 min each. Bacterial load of scraped biofilm was determined by the drop plate method.

\section{Biofilm development}

K. pneumoniae biofilm was grown in 96-well microtiter plate by a modification of an established method of Bedi et al. ${ }^{12}$ Briefly, wells of microtiter plate were inoculated with $50 \mu \mathrm{l}$ of soyabean casein digest medium and $50 \mu \mathrm{l}$ of bacterial culture $\left(\mathrm{OD}_{600}=0.3\right)$ equivalent to $10^{8} \mathrm{CFU} \mathrm{ml}{ }^{-1}$ of $\mathrm{K}$. pneumoniae and incubated at $37^{\circ} \mathrm{C}$ overnight. In each test, control wells containing sterile nutrient broth were included as plate sterility control. After incubation, planktonic cells in the fluid were removed and wells were washed thoroughly three times with NS. The biofilm matrix was then scraped with a sterile pipette tip, suspended in NS and vortexed for $3 \mathrm{~min}$. Microbial load of biofilm was enumerated by viable cell counting.

For the establishment of 7-day old biofilm, spent medium was replaced with fresh sterile nutrient broth in rest of the wells and plate was reincubated at $37^{\circ} \mathrm{C}$ overnight. Media in each well were replaced every $24 \mathrm{~h}$ until the seventh day of the experiment. Every day, wells were stained with $0.1 \%$ crystal violet for $10 \mathrm{~min}$. Following the staining step, the colorant was discarded and the wells were rinsed three times with NS to remove excess stain. Then, the dye incorporated by the biofilm or present in the control wells was solubilized in absolute ethanol. The OD of the stained biofilm and control wells was read at $595 \mathrm{~nm}$ using enzyme-linked immunosorbent assay reader (BIORAD iMark Microplate Reader, Bio-Rad Laboratories, Inc., Hercules, CA, USA). Microbial load of the biofilm was also enumerated every day by viable cell count till 7 days.

Biofilm was also developed on glass coverslips $(18 \mathrm{~mm} \times 18 \mathrm{~mm}$; 0.08-0.12 mm; Corning Glass, Corning, NY, USA). Biofilm on glass coverslips was grown by the Tip-box batch culture method of Hughes et al. ${ }^{13}$ Biofilms were allowed to grow at air-liquid interface. Tip-box mounted coverslips and the nutrient broth were sterilized separately. A measure of $100 \mu$ of bacterial culture $\left(10^{8} \mathrm{CFU} \mathrm{ml}{ }^{-1}\right)$ was added to the broth, which was then poured into the tip box. The whole setup was incubated at $37^{\circ} \mathrm{C}$. The desired number of coverslips were removed and used. Spent growth medium in the culture box was replaced every $24 \mathrm{~h}$.

Colony biofilms of K. pneumoniae were grown on black, polycarbonate disc (EMD Millipore Corporation, Billerica, MA, USA) according to the method described by Anderl et al..$^{14}$ with modifications. Briefly, culture of K. pneumoniae was grown overnight in nutrient broth at $37{ }^{\circ} \mathrm{C}$ and diluted to an $\mathrm{OD}(600 \mathrm{~nm}, 1-\mathrm{cm}$ path length) of 0.300 in normal saline. A measure of $10 \mu \mathrm{l}$ of diluted planktonic culture was used to inoculate individual sterile, black, polycarbonate membrane filters (diameter, $47 \mathrm{~mm}$; pore size, $0.2 \mu \mathrm{m}$ ) resting on agar culture medium. The membranes were sterilized by UV exposure ( $15 \mathrm{~min}$ per side) before inoculation. The plates were inverted and incubated at $37^{\circ} \mathrm{C}$ till 7 days, with the membrane-supported biofilms transferred to fresh culture medium every $24 \mathrm{~h}$. The morphology and diameter of colony biofilm was recorded till 7 days.

\section{Susceptibility tests}

MIC of planktonic cells at mid-log phase. The MIC of the planktonic cells was determined in accordance with Clinical and Laboratory Standards Institute (formerly NCCLS) guidelines. ${ }^{15}$ Antibiotics were used at a concentration range of $1024-0.062 \mu \mathrm{g}^{-1}$. The highest dilution of the antibiotic showing visible inhibition of bacterial growth after overnight incubation at $37^{\circ} \mathrm{C}$ was taken as the MIC of the drug.

MBC of planktonic cells at mid-log phase. The MBC of the antibiotics against planktonic bacteria at mid-log phase was determined by plating the contents of the tubes containing MICs and higher concentrations of the antibiotics on nutrient agar and incubated at $37^{\circ} \mathrm{C}$ for $24 \mathrm{~h}$. Before plating, the bacteria in antibiotic-treated and control tube were pelleted by centrifugation at 10000 r.p.m. for $10 \mathrm{~min}$, washed two times with NS and finally suspended in $1 \mathrm{ml} \mathrm{NS}$. The resulting cell suspension was serially diluted, and the viable count was enumerated by drop plating. ${ }^{16}$

MIC of adherent monolayer. To examine the MIC of single antibiotic for adherent monolayer, $100 \mu \mathrm{l}$ volume of twofold serial dilutions of antibiotics, ranging from 1024 to $0.062 \mu \mathrm{g} \mathrm{ml}^{-1}$, in Tryptose Soy Broth were added to microwells with established adherent monolayer. Plates were incubated at $37^{\circ} \mathrm{C}$ overnight. MIC of adherent monolayer was defined as the lowest concentration of antibiotics that prevented the appearance of visible turbidity after overnight exposure.

$M B C$ of adherent monolayer. For the determination of MBC, the contents of the wells containing MICs and higher concentrations of the antibiotics were removed. To the emptied wells, $100 \mu \mathrm{l}$ NS was added and contents were aspirated using a micropipette to remove any loosely bound bacteria. The procedure of washing was repeated three times and cells adhered to the well surface was scraped using a sterile spatula. Scraped biofilm was suspended in $100 \mu \mathrm{l}$ NS. Suspended biofilm was vortexed three times for $1 \mathrm{~min}$ each. Viable cell count was estimated by plating appropriate dilutions onto nutrient agar. The log reduction for adherent monolayers was calculated by dividing the pre-determined viable count in the untreated wells by the viable counts in individual wells after antibiotic exposure. The monolayer MBC was defined as the lowest concentration of antibiotic that reduced bacterial numbers by 3 logs.

MIC of biofilm-grown bacteria. MIC of antibiotic at stationary phase could not be tested by standard procedure because of high cell density of bacteria in the overnight culture. The method of Ceri et al..$^{6}$ was used to determine MIC of biofilm-grown bacteria. Biofilm established in 96-well microplate was exposed to $100 \mu \mathrm{l}$ of twofold serial dilutions of antibiotics ranging from 1024 to $0.062 \mu \mathrm{g} \mathrm{ml}^{-1}$ in Tryptose Soy Broth. The microplate was then incubated at $37^{\circ} \mathrm{C}$ for $24 \mathrm{~h}$. After overnight challenge, the supernatant from each well was carefully transferred to the wells in a new 96-well microplate without disturbing the biofilm, and the turbidity of the contents was visually assessed. The biofilm MIC was defined as the lowest concentration of antibiotic at which no visible growth was observed. When exposed to antibiotics at or above this concentration, the biofilm shed bacteria showed susceptibility to antibiotic.

MBC of biofilm cells. MBCs of the biofilm bacteria were also assessed on the basis of reduction in viable counts after overnight exposure to antibiotics. The viable bacteria remaining in biofilm in wells containing MICs or higher concentrations of antibiotic were counted by plating on nutrient agar and incubated at $37^{\circ} \mathrm{C}$ for $24 \mathrm{~h}$. MBC of biofilm was calculated by dividing the predetermined viable count of untreated biofilm by viable counts obtained after antibiotic exposure. 
MBC of planktonic cells at stationary phase. The MBC of antibiotics against planktonic bacteria at stationary phase was determined following the Clinical and Laboratory Standards Institute guidelines, ${ }^{17}$ except that stationary phase cultures replaced log-phase cultures. The MBCs for planktonic cells at stationary phase and for biofilms were defined as the minimum concentration of antibiotics required to reduce bacterial numbers by at least 3 logs.

Effect of antibiotics on preformed biofilms. As the maximum clinically achievable concentrations of amikacin and ciprofloxacin are 40 and $4.5 \mu \mathrm{g} \mathrm{ml}^{-1}$, respectively ${ }^{18}$ (intravenously), the concentrations used in the susceptibility assay were $20,40,60$ and $80 \mu \mathrm{g} \mathrm{ml}^{-1}$ for amikacin and 2, 4, 6 and $8 \mu \mathrm{g} \mathrm{ml}^{-1}$ for ciprofloxacin. The microtiter plates were incubated at $37^{\circ} \mathrm{C}$ for 7 days. The control biofilms and antibiotic-treated biofilms were sampled every $24 \mathrm{~h}$ and viable bacteria were enumerated by drop plating. ${ }^{16}$ Experiments were performed in duplicate.

Evaluation of polysaccharide matrix. The polysaccharide component of the biofilm formed on the coverslip was stained for $15 \mathrm{~min}$ in dark using calcofluor staining solution ( $300 \mu \mathrm{g} \mathrm{ml}^{-1}$; Sigma-Aldrich Chemicals, St Louis, MO, USA) and coverslips were observed under fluorescent microscope (Olympus America Inc., Center Valley, PA, USA).

Scanning electron microscopy of colony biofilm. Colony biofilm was examined by scanning electron microscopy (SEM) according to the method of Yasuda et al. ${ }^{19}$ with modifications. Briefly, the colony biofilms were fixed with $2.5 \%$ glutaraldehyde $(\mathrm{pH} 7.2)$ at room temperature for $1 \mathrm{~h}$, washed three times in the rinsing buffer (Sorensen's phosphate buffer $\left(0.1\right.$ moll $\left.^{-1}, \mathrm{pH} 7.2\right)+7 \%$ sucrose) at $4{ }^{\circ} \mathrm{C}$ for $15 \mathrm{~min}$ each, and fixed with $1 \%$ osmium tetraoxide at $4{ }^{\circ} \mathrm{C}$ for $30 \mathrm{~min}$. This was followed by three washings in rinsing buffer at $4{ }^{\circ} \mathrm{C}$ for $15 \mathrm{~min}$ each and dehydration of the biofilms in a series of ethanol solutions (50\%, $10 \mathrm{~min} ; 70 \%, 10 \mathrm{~min} ; 80 \%, 15 \mathrm{~min} ; 90 \%, 15 \mathrm{~min}$; and $100 \%, 20 \mathrm{~min}$ ). The specimens were then critical point dried, coated with gold and observed under SEM (Jeol JSM-6100, JEOL Ltd., Tokyo, Japan).

\section{Statistical analysis}

All the experiments were performed in duplicate and repeated at least three times. The mean and s.d. were calculated from the results. The bacterial count was converted to $\log _{10}$. The effect of different treatments on biofilm eradication was evaluated by two-way analysis of variance and Student's t-test and $P<0.01$ was considered significant.

\section{RESULTS}

\section{Biofilm formation}

Figure 1 shows the quantitative results (mean \pm s.d. OD $_{595}$ ) of crystal violet staining of biofilm formed by K. pneumoniae. It showed an increase in biofilm formation, with a peak on the fifth day of incubation, after which the biofilm formation decreased progressively.



Figure 1 Biofilm formation by Klebsiella pneumoniae for 7 days as determined by crystal violet staining $\left(\mathrm{OD}_{595}\right)$ and biomass quantification. $\mathrm{CV}$, coefficient of variation.
The results of this semiquantitative method were also confirmed by biomass quantification.

\section{Antimicrobial susceptibilities of $K$. pneumoniae grown in different modes}

Comparison of adherent monolayers with planktonic cultures at mid-log phase. There were no significant differences in antibiotic susceptibility pattern of planktonic cells at mid-log phase and adherent monolayers as determined by MIC. However, MBC of amikacin was found to be eight times higher for monolayer grown K. pneumoniae than planktonic cells. The MBC of piperacillin was $>1024 \mu \mathrm{g} \mathrm{ml}^{-1}$ for adherent monolayers, which is well beyond the highest achievable serum concentrations (Table 1 ).

Biofilms compared with planktonic cultures at mid-log phase and adherent monolayers. Differences in susceptibilities of biofilm-grown cells and planktonic cells at mid-log phase were antibiotic dependent. The MICs of amikacin and ciprofloxacin for biofilm cells were found to be two and four times higher than that of log planktonic cells. However, the MIC of cell wall active antibiotic was significantly higher for biofilm cells than for planktonic cells at mid-log phase; that is, eight times for piperacillin. The MBCs of all the antibiotics were significantly higher for biofilm cells than planktonic cells at mid-log phase. Adherent monolayers showed similar trend as was seen with log planktonic cultures.

Biofilms compared with planktonic cultures at stationary phase. The MBC of piperacillin was above the upper limit of detection $\left(\geqslant 1024 \mu \mathrm{g} \mathrm{ml}^{-1}\right)$ when checked against planktonic cells at stationary phase or in biofilm mode. There was no significant difference in MBCs of amikacin and ciprofloxacin between biofilms and planktonic cultures at stationary phase.

Dose-dependent effect of antibiotics on preformed biofilm. The effect of amikacin and ciprofloxacin on preformed biofilm was studied and the results are presented in Figures 2 and 3. Two-way analysis of variance showed that amikacin and ciprofloxacin were most effective at 40 and $6 \mu \mathrm{g} \mathrm{ml}^{-1}$, respectively. Treatment of biofilm using amikacin at $40 \mu \mathrm{g} \mathrm{ml}^{-1}$ and ciprofloxacin at $6 \mu \mathrm{g} \mathrm{ml}^{-1}$ led to significant reduction of 2.02 and 2.18 logs in the bacterial count $(P<0.01)$. This effect was uniformly seen in 1- to 4-day-old biofilms, indicating that amikacin was effective against younger biofilm at $40 \mu \mathrm{g} \mathrm{ml}^{-1}$, which is also the highest achievable serum concentration. Similarly, ciprofloxacin showed effectiveness for young biofilm at $6 \mu \mathrm{g} \mathrm{ml}^{-1}$, which is more than the achievable concentration $\left(4.5 \mu \mathrm{g} \mathrm{ml}^{-1}\right)$ in the

Table 1 Antimicrobial activity of three antibiotics against K. pneumoniae B5055 in different phases of biofilm formation.

\begin{tabular}{|c|c|c|c|c|c|c|c|}
\hline \multirow[b]{2}{*}{ Mode of growth } & \multirow{2}{*}{ 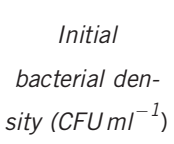 } & \multicolumn{2}{|c|}{$\begin{array}{c}\text { Ciprofloxacin } \\
\left(\mu g \mathrm{ml}^{-1}\right)\end{array}$} & \multicolumn{2}{|c|}{$\begin{array}{l}\text { Amikacin } \\
\left(\mu g m l^{-1}\right)\end{array}$} & \multicolumn{2}{|c|}{$\begin{array}{c}\text { Piperacillin } \\
\left(\mu g m l^{-1}\right)\end{array}$} \\
\hline & & $M I C$ & $M B C$ & $M I C$ & $M B C$ & $M I C$ & $M B C$ \\
\hline Log planktonic & $6.2 \times 10^{6}$ & 0.5 & 0.5 & 16 & 16 & 16 & 128 \\
\hline Adherent monolayer & $8.8 \times 10^{6}$ & 0.25 & 2 & 16 & 128 & 16 & $>1024$ \\
\hline Stationary Planktonic & $2.7 \times 10^{8}$ & - & 512 & - & 256 & - & $>1024$ \\
\hline Mature Biofilm & $3.2 \times 10^{8}$ & 2 & 512 & 32 & 512 & 128 & $>1024$ \\
\hline
\end{tabular}




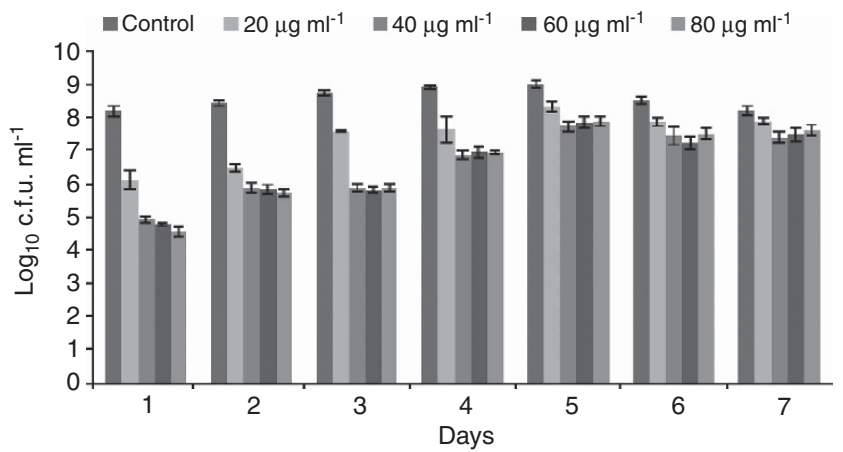

Figure 2 Susceptibility of Klebsiella pneumoniae biofilm to different concentrations of amikacin. Experiment was performed in duplicate. Error bars indicate s.d.

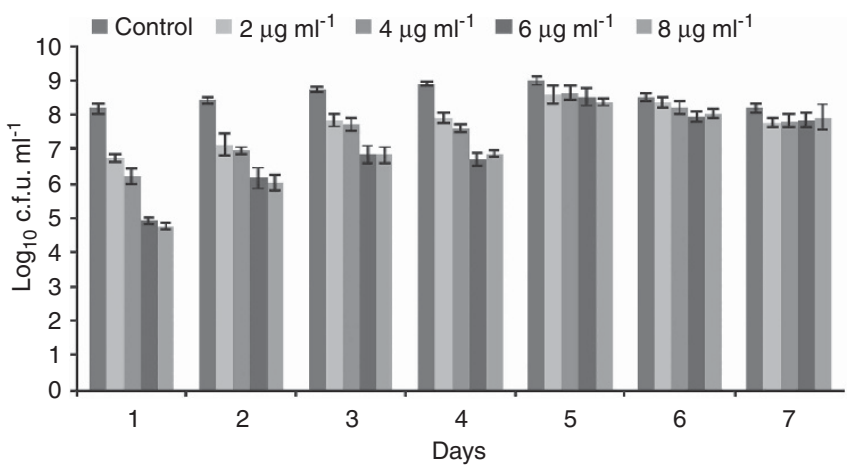

Figure 3 Susceptibility of Klebsiella pneumoniae biofilm to different concentrations of ciprofloxacin. Experiment was performed in duplicate. Error bars indicate s.d.

serum. Both antibiotics were ineffective against older biofilm (that is, fifth day onwards) as insignificant reduction in the log bacterial count $(P>0.01)$; that is, 0.28 and 0.59 for ciprofloxacin and amikacin, respectively, was seen, even at concentrations two times the highest achievable serum concentration.

Microscopic analysis of biofilms on coverslips. The biofilm stained with calcofluor white revealed slime production both in young and old biofilms (Figure 4). However, comparison of Figures $4 \mathrm{a}$ and $\mathrm{b}$ indicates that bacteria in older biofilms were more heavily encased in extracellular polysaccharide matrix than bacteria in young biofilm.

Morphological characteristics of biofilm. Both horizontal and vertical development of biofilm was observed during a 7-day period. There was significant increase in diameter of colony biofilm from 5 to $14 \mathrm{~mm}$ over a period of 7 days forming thick bacterial masses. Figure 5 clearly shows the change in colony diameter and colony morphology with increase in biofilm age. Young biofilm was flat with depression in the center, shiny and mucoid in appearance (Figure 5a), whereas older biofilm was centrally elevated, large in size and show light source reflecting off the edge of a shiny layer of slime extending beyond the edges of the colony (Figure 5b). The biofilms developed on polycarbonate discs were also observed using SEM. SEM images of biofilm on polycarbonate discs after sample fixation and dehydration indicated biofilm formation on the membrane surfaces with significant extracellular polymeric substance (EPS) production (Figure 6).
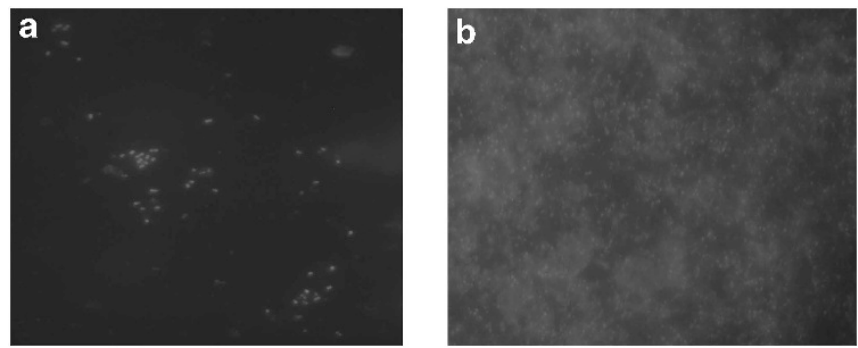

Figure 4 Fluorescent microscopy of biofilm stained with calcofluor white. The blue clouds represent concentrations of extracellular polysaccharide secreted by bacteria in (a) young biofilm (b) old biofilm. A full color version of this figure is available at The Journal of Antibiotics journal online.


Figure 5 Colony morphology of (a) young and (b) old biofilm of Klebsiella pneumoniae grown on black polycarbonate membrane. A full color version of this figure is available at The Journal of Antibiotics journal online.

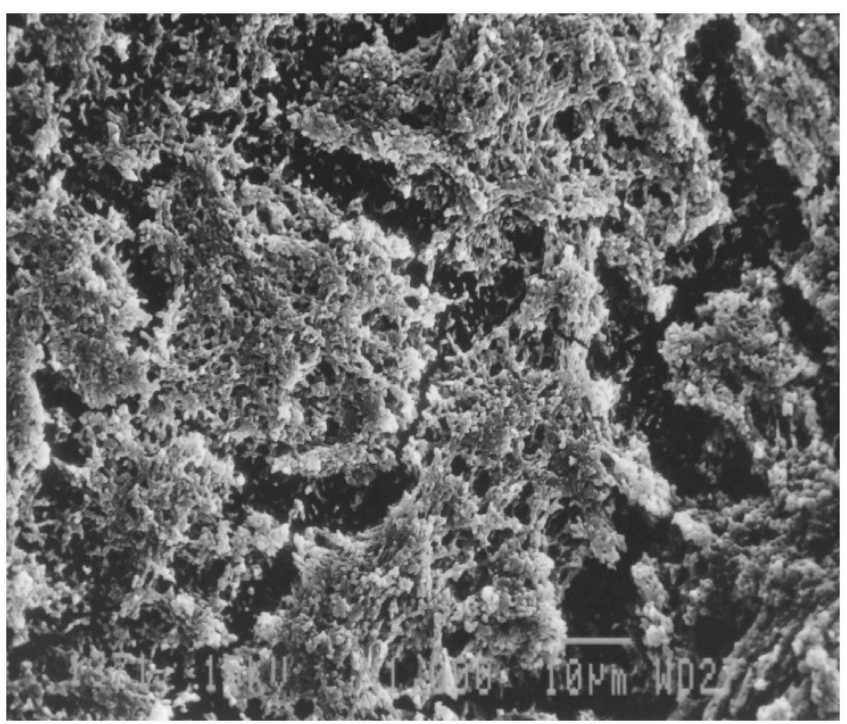

Figure 6 Representative SEM image of the Klebsiella pneumoniae biofilm showing significant EPS production.

\section{DISCUSSION}

The MIC has been used as a gold standard for the determination of antimicrobial susceptibility of planktonic organisms. It serves as an important reference in the treatment of many acute infections. ${ }^{20,21} \mathrm{An}$ antibiotic is considered to be clinically irrelevant if it is found to be ineffective using the MIC assay. ${ }^{22}$ Moreover, an organism that is 
sensitive in vitro may not be effective in vivo, ${ }^{22-26}$ probably due to the presence of bacterial biofilms, especially in chronic or device-related infections. It has been revealed that in nature, more than $99 \%$ of all bacteria exist in biofilm mode. ${ }^{27}$ Hence, clinicians fail to eradicate these cells as concentrations of antibiotics much higher than the MICs are required to eradicate bacteria in biofilms. In this study, an attempt was made to describe a system that may help in elucidating the effectiveness of different classes of antibiotics in the eradication of bacteria in biofilms. This was carried out by comparing the susceptibility of bacteria in different phases of growth, relevant to biofilm formation.

Planktonic cultures at mid-log phase, adherent monolayers, mature biofilms and planktonic cultures at stationary phase were assessed for their susceptibility to different classes of antibiotics. The rationale was to compare the susceptibilities of biofilm-grown cells and planktonic cells of the same cell density. In this study, we found that MIC of planktonic cells at mid-log phase and adherent monolayer was similar. These results are consistent with those reported by Labthavikul et al., ${ }^{28}$ who also found similar MIC and MBC values for organisms in this mode of growth. MBC of amikacin was found to be eight times higher and that of piperacillin $>1024 \mu \mathrm{g} \mathrm{ml}^{-1}$ for adherent monolayers, which is well beyond the highest achievable serum concentration. This is in agreement with the results of other studies where significantly high $\mathrm{MBC}$ values for adherent form than the planktonic form of Staphylococcus aureus and Pseudomonas aeruginosa have been reported. ${ }^{29,11}$ This difference is likely to be due to the different kinetics of biofilm formation by S. epidermidis and $P$. aeruginosa.

When biofilm cells were compared with planktonic culture at midlog phase and adherent monolayer level, the MICs of amikacin and ciprofloxacin for biofilm cells were found to be two and four times higher than the log planktonic cells. On the contrary, the MIC of piperacillin (cell wall active) was significantly higher (eight times) than these two antibiotics. Similarly, the MBC of all the antibiotics was significantly higher for biofilm cells than planktonic cells at midlog phase. Adherent monolayers also showed similar trend as observed with log planktonic culture. Other researchers have also reported that MICs of $\beta$-lactam antibiotics for biofilm bacteria were much higher than the conventional MICs for $P$. aeruginosa and S. aureus planktonic forms. ${ }^{7,30}$ Abdi-Ali et al. ${ }^{31}$ have found that $\beta$-lactam antibiotics showed hardly any bactericidal activity against the biofilm cells, whereas other antibiotics showed bactericidal activity, although at higher concentrations. One of the factors responsible for biofilm tolerance is the slow growth because of poor access to essential nutrients to the cells that are embedded deep within the matrix. A phenomenon known as phenotypic tolerance is widely recognized, in which bacteria deprived of an essential nutrient soon develop resistance to lysis by $\beta$-lactam antibiotics. $^{32,33}$ In addition, the cells that are released from the biofilm are likely to be less active than planktonic cells at mid-log phase, probably because they have entered in a free-living mode more recently. These are more similar to cells at lag-phase of growth, and require a change in gene expression to adapt to the new environment. Cell wall active antibiotics, such as $\beta$-lactams, require rapid bacterial growth to kill cells because penicillin binding proteins are poorly expressed in slow-growing bacteria. Thus, piperacillin was found to be least effective for biofilm cells. There was also no significant difference in the MBC of all antibiotics for biofilms and planktonic cultures at stationary phase. This is consistent with the observation of Spoering and Lewis, ${ }^{34}$ according to whom bacteria within biofilms are considered more close to stationary phase cultures, ${ }^{35,36}$ a factor believed to be responsible for the insusceptibility of biofilms to antibiotics. $^{37}$

The role of biofilm formation and development by bacteria has been suggested to be an important stage in the pathogenesis of numerous bacterial infections. ${ }^{38-40}$ The establishment of biofilm by pathogenic bacteria on the tissues of susceptible hosts is believed to inhibit the effectiveness of antibiotic treatment, protect against host defense mechanisms and facilitate bacterial communication, leading to the expression of virulence determinants. ${ }^{1}$ Biofilm age is also a decisive factor in determining the outcome of a therapeutic regimen. ${ }^{41}$ Older biofilms are known to resist higher antibiotic concentrations than planktonic cells as well as younger biofilms. ${ }^{42}$ Hence, in this study, the effect of ciprofloxacin and amikcin at the highest achievable serum concentration on preformed biofilms was checked. The results showed that amikacin was effective against younger biofilm (that is, upto 4-day-old biofilm) at $40 \mu \mathrm{g} \mathrm{ml}^{-1}$, which is also the highest achievable serum concentration, whereas ciprofloxacin showed effectiveness for young biofilm at $6 \mu \mathrm{g} \mathrm{ml}^{-1}$, which is more than the highest serum achievable concentration; that is, $4.5 \mu \mathrm{g} \mathrm{ml}^{-1}$. Both antibiotics were ineffective against older biofilm (that is, fifth day onwards). This might be due to inaccessibility of bacteria to antibiotics as diffusion of ciprofloxacin through $P$. aeruginosa biofilm took considerably longer time ( $>20 \mathrm{~min}$ ) than its diffusion through the sterile surface $(40 \mathrm{~s}) .{ }^{43}$

The phenomenon of limited penetration of some antibiotics into biofilms is postulated to be due to the presence of EPS or 'matrix'. The latter functions as a chemical retardant to allow survival of bacteria in the presence of antibiotics. Different organisms produce differing amounts of EPS, and the amount of EPS increases with the age of the biofilm. ${ }^{44}$ This study visually demonstrates the increased production of polysaccharide component of matrix in older biofilm compared with younger biofim using calcofluor white stain. This in turn might be responsible for increased resistance of older biofilm of $K$. pneumoniae to amikacin and ciprofloxacin as observed in this study. SEM analysis of colony biofilm revealed three-dimensional structure as well as EPS production, which could be helpful for analyzing the structure of biofilm.

This study gives more realistic information about the concentration of different antibiotics required to eradicate the biofilm cells of K. pneumoniae. Of the antibiotics tested, amikacin appeared to be most effective drug to treat young biofilm as inhibition of biofilm of K. pneumoniae was observed at the effective achievable concentration. The results of this study also suggest that early initiation of treatment with antibiotics of the bacteria in biofilm mode is essential as the biofilm grows older; there is increased production of extracellular polysaccharide making the older biofilms resistant to antibiotics. However, the data generated in this study is based on the observations made on a standard strain of $K$. pneumoniae. It is suggested that future studies should concentrate on wild-type isolates so as to have more meaningful data.

\section{CONFLICT OF INTEREST}

The authors declare no conflict of interest.

\section{ACKNOWLEDGEMENTS}

This work was supported by funding from the Department of Science and Technology (DST), India, in the form of a research fellowship. We thank Mrs Rajni of the Postgraduate Institute of Medical Education and Research (PGIMER), Chandigarh, India, for assistance in the operation of fluorescent microscope. 
1 Lavender, H. F., Jagnow, J. R. \& Clegg, S. Biofilm formation in vitro and virulence in vivo of mutants of Klebsiella pneumoniae. Infect. Immunity 72, 4888-4890 (2004).

2 Moscoso, M., García, E. \& López, R. Pneumococcal biofilms. Int. Microbiol. 12, 77-85 (2009).

3 Ceri, H., Olson, M. E., Morck, D. W., Read, R. R. \& Buret, A. G. The Calgary Biofilm Device: measurement of antimicrobial sensitivity of bacterial biofilms. J. Clin. Microbiol. 37, 1771-1776 (1999).

4 Gilbert, P., McBain, A. \& Rickard, A. H. in Multiple drug resistant bacteria. (eds, Amábile-Cuevas, C. F.) 81-107, (Horizon Scientific Press, London, UK, 2003).

5 Wentland, E. J., Stewart, P. S., Huang, C. T. \& McFeters, G. A. Spatial variations in growth rate within Klebsiella pneumoniae colonies and biofilm. Biotechnol. Prog. 12, 316-321 (1996).

6 Ceri, H. et al. The MBEC Assay System: multiple equivalent biofilms for antibiotic and biocide susceptibility testing. Methods Enzymol. 337, 377-385 (2001).

7 Moskowitz, S. M., Foster, J. M., Emerson, J. \& Burns, J. L. Clinically feasible biofilm susceptibility assay for isolates of Pseudomonas aeruginosa from patients with cystic fibrosis. J. Clin. Microbiol. 42, 1915-1922 (2004).

8 Pettit, R. K. et al. Microplate Alamar blue assay for Staphylococcus epidermidis biofilm susceptibility testing. Antimicrob. Agents Chemother. 49, 2612-2617 (2005).

9 Cheung, G. Y. \& Otto, M. Understanding the significance of Staphylococcus epidermidis bacteremia in babies and children. Curr. Opin. Infect. Dis. 23, 208-216 (2010).

10 Qu, Y., Istivan, T. S., Daley, A. J., Rouch, D. A. \& Deighton, M. A. Comparison of various antimicrobial agents as catheter lock solutions: preference for ethanol in eradication of coagulase-negative staphylococcal biofilms. J. Med. Microbiol. 58, 442-450 (2009).

11 Miyake, Y., Fujiwara, S., Usui, T. \& Suginaka, H. Simple method for measuring the antibiotic concentration required to kill adherent bacteria. Chemotherapy $\mathbf{3 8}$, 286-290 (1992)

12 Bedi, M. S., Verma, V. \& Chhibber, S. Amoxicillin and specific bacteriophage can be used together for eradication of biofilm of Klebsiella pneumoniae B5055. World J. Microbiol. Biotechnol. 25, 1145-1151 (2009).

13 Hughes, K. A., Sutherland, I. W., Clark, J. \& Jones, M. V. Biofilm susceptibility to bacteriophage attack: the role of phage-borne polysaccharide depolymerase. Microbiology 144, 3039-3047 (1998).

14 Anderl, J. N., Franklin, M. J. \& Stewart, P. S. Role of antibiotic penetration limitation in Klebsiella pneumoniae biofilm resistance to ampicillin and ciprofloxacin. Antimicrob. Agents Chemother. 44, 1818-1824 (2000).

15 Clinical and Laboratory Standards Institute. Performance Standards for Antimicrobial Susceptibility Testing: Sixteenth Informational Supplement M100-S16 (CLSI, Wayne, PA, USA, 2006).

16 Miles, A. A., Misra, S. S. \& Irwin, J. O. The estimation of the bactericidal power of the blood. J. Hyg. (Lond) 38, 732-749 (1938).

17 Clinical and Laboratory Standards Institute. Methods for Dilution Antimicrobial Susceptibility Tests for Bacteria that Grow Aerobically-. 7th edn, Approved standard M7-A7 (CLSI, Wayne, PA, USA, 2004).

18 Yao, J. D. C. \& Moellering, Jr. R. C. in Manual of Clinical Microbiology Antibacterial Agents. (eds, Murray, P. R., Baron, E. J., Pfaller, M. A., Jorgensen, J. H. \& Yolken, R. H. 1039-1073 (American Society for Microbiology, Washington, DC, USA, 2003).

19 Yasuda, H., Ajiki, Y., Koga, T. \& Yokota, T. Interaction between clarithromycin and biofilms formed by Staphylococcus epidermidis. Antimicrob. Agents Chemother. 38, 138-141 (1994).

20 Costerton, J. W., Lewandowski, Z., Caldwell, D. E., Korber, D. R. \& Lappin-Scott, H. M. Microbial biofilms. Ann. Rev. Microbiol. 49, 711-745 (1995).

21 Prescott, J. F. \& Baggott, J. D. Antimicrobial susceptibility testing and antimicrobial drug dosage. J. Am. Vet. Med. Assoc. 187, 363-368 (1995).

22 Langston, V. C. in Current Veterinary Therapy. 4: Food Animal Practice. Antimicrobial Use in Food Animals (eds, Howard, J. L. \& Smith, R. A.) 17-32 (WB Saunders, Philadelphia, USA, 1999).
23 Owens, W. E., Ray, C. H., Watts, J. L. \& Yancey, R. J. Comparison of success of antibiotic therapy during lactation and results of antimicrobial susceptibility tests for bovine mastitis. J. Dairy Sci. 80, 313-317 (1997).

24 Sandholm, M., Kaartinen, L. \& Pyorala, S. Bovine mastitis-Why does antibiotic therapy not always work? An overview. J. Vet. Pharmacol. Ther. 13, 248-260 (1990).

25 Potera, C. Forging a link between biofilms and disease. Science 283, 1837-1838 (1999).

26 Pratt, L. A. \& Kolter, R. Genetic analysis of biofilm formation. Curr. Opin. Microbiol. 2, 598-603 (1999).

27 Costerton, J. W. et al. Bacterial biofilms in nature and disease. Annu. Rev. Microbiol. 41, 435-464 (1987).

28 Labthavikul, P., Petersen, P. J. \& Bradford, P. A. In vitro activity of tigecycline against Staphylococcus epidermidis growing in an adherent-cell biofilm model. Antimicrob. Agents Chemother. 47, 3967-3969 (2003).

29 Aaron, S. D., Ferris, W., Ramotar, K., Vandemheen, K. \& Chan, F. Single and combination antibiotic susceptibilities of planktonic, adherent, and biofilm-grown Pseudomonas aeruginosa isolates cultured from sputa of adults with cystic fibrosis. J. Clin. Microbiol. 40, 4172-4179 (2002).

30 Melchior, M. B., Fink-Gremmels, J. \& Gaastra, W. Comparative assessment of the antimicrobial susceptibility of Staphylococcus aureus isolates from bovine mastitis in biofilm versus planktonic culture. J. Vet. Med. B 53, 326-332 (2006).

31 Abdi-Ali, A., Mohammadi-Mehr, M. \& Agha Alaei, Y. Bactericidal activity of various antibiotics against biofilm-producing Pseudomonas aeruginosa. Int. J. Antimicrob. Agents 27, 196-200 (2006).

32 Tuomanen, E., Cozens, R., Tosch, W., Zak, O. \& Tomasz, A. The rate of killing of Escherichia coli by $\beta$-lactam antibiotics is strictly proportional to growth rate. J. Gen. Microbiol. 132, 1297-1304 (1986).

33 Tuomanen, E. \& Tomasz, A. Mechanism of phenotypic tolerance of non-growing pneumococci to $\beta$-lactam antibiotics. Scand. J. Infect. Dis. 74 102-112 (1991).

34 Spoering, A. L. \& Lewis, K. Biofilms and planktonic cells of Pseudomonas aeruginosa have similar resistance to killing by antimicrobials. J. Bacteriol. 183, 6746-6751 (2001).

35 Evans, R. C. \& Holmes, C. J. Effect of vancomycin hydrochloride on Staphylococcus epidermidis biofilm associated with silicone elastomer. Antimicrob. Agents Chemother. 31, 889-894 (1987).

36 Prosser, B., Taylor, D., Dix, B. A. \& Cleeland, R. Method of evaluating effects of antibiotics upon bacterial biofilms. Antimicrob. Agents Chemother. 31, 1502-1506 (1987).

37 Brown, M. R. W., Allison, D. G. \& Gilbert, P. Resistance of bacterial biofilms to antibiotics: a growth-rate related effect? J. Antimicrob. Chemother. 22, 777-783 (1988).

38 Costerton, J. W., Stewart, P. S. \& Greenberg, E. P. Bacterial biofilms: a common cause of persistent infections. Science 284, 1318-1322 (1999).

39 Donlan, R. M. Biofilm formation: a clinically relevant microbiological process. Clin. Infect. Dis. 33, 1387-1392 (2001).

40 Watnick, P. \& Kolter, R. Biofilm, city of microbes. J. Bacteriol. 182, 2675-2679 (2000).

41 Sedlacek, M. J. \& Walker, C. Antibiotic resistance in an in vitro subgingival biofilm model. Oral Microbiol. Immunol. 22, 333-339 (2007).

42 Ito, A., Taniuchi, A., May, T., Kawata, K. \& Okabe, S. Increased antibiotic resistance of Escherichia coli in mature biofilms. Appl. Environ. Microbiol. 75, 4093-4100 (2009).

43 Suci, P. A., Mittleman, M. W., Yu, F. P. \& Geesey, G. G. Investigation of ciprofloxacin penetration into Pseudomonas aeruginosa biofilms. Antimicrob. Agents Chemother. $\mathbf{3 8}$ 2125-2133 (1994).

44 Leriche, V., Sibille, P. \& Carpentier, B. Use of an enzyme-linked lectinsorbent assay to monitor the shift in polysaccharide composition in bacterial biofilms. Appl. Environ. Microbiol. 66, 1851-1856 (2000). 